\title{
Coupled-channels analyses for large-angle quasi-elastic scattering in massive systems
}

\author{
Muhammad Zamrun F. and K. Hagino \\ Department of Physics, Tohoku University, Sendai 980-8578, Japan
}

S. Mitsuoka and H. Ikezoe

Advanced Science Research Center, Japan Atomic Energy Agency, Tokai, Ibaraki 319-1195, Japan

(Dated: November 16, 2018)

\begin{abstract}
We discuss in detail the coupled-channels approach for the large-angle quasi-elastic scattering in massive systems, where many degrees of freedom may be involved in the reaction. We especially investigate the effects of single, double and triple phonon excitations on the quasi-elastic scattering for ${ }^{48} \mathrm{Ti},{ }^{54} \mathrm{Cr},{ }^{56} \mathrm{Fe},{ }^{64} \mathrm{Ni}$ and ${ }^{70} \mathrm{Zn}+{ }^{208} \mathrm{~Pb}$ systems, for which the experimental cross sections have been measured recently. We show that the present coupled-channels calculations well account for the overall width of the experimental barrier distribution for these systems. In particular, it is shown that the calculations taking into account single quadrupole phonon excitations in ${ }^{48} \mathrm{Ti}$ and triple octupole phonon excitations in ${ }^{208} \mathrm{~Pb}$ reasonably well reproduce the experimental quasi-elastic cross section and barrier distribution for the ${ }^{48} \mathrm{Ti}+{ }^{208} \mathrm{~Pb}$ reaction. On the other hand, ${ }^{54} \mathrm{Cr},{ }^{56} \mathrm{Fe},{ }^{64} \mathrm{Ni}$ and ${ }^{70} \mathrm{Zn}+{ }^{208} \mathrm{~Pb}$ systems seem to require the double quadrupole phonon excitations in the projectiles in order to reproduce the experimental data.
\end{abstract}

PACS numbers: 24.10.Eq, 25.60.Pj, 25.70.Bc, 27.80.+w

\section{INTRODUCTION}

It is now well established that the internal structure of colliding nuclei strongly influences heavy-ion collisions at energies around the Coulomb barrier. In particular, the coupling to the collective excitations (rotation and vibrational states) in the target and projectile nuclei participating in the reaction significantly enhances the fusion cross sections for intermediate mass systems [1, 2]. Such couplings give rise to a distribution of the Coulomb barrier 1, 2, 3], which can most easily be visualized for reactions involving a deformed nucleus. In this case, the nucleus-nucleus potential depends on the orientation angle of the deformed nucleus with respect to the beam direction. Since the orientation angle distributes isotropically at the initial stage of the reaction, so does the potential barrier. The concept of barrier distribution can be extended also to systems with a non-deformed target [3], where the distribution originates from the coupling between the relative motion and vibrational excitations in the colliding nuclei and/or transfer processes. Notice that, although this concept is exact only when the excitation energy is zero, to a good approximation it holds also for systems with a non-zero excitation energy [4, 5].

In Ref. [6], Rowley et al. have argued that the barrier distribution can be directly extracted from a measured fusion cross section $\sigma_{\text {fus }}(E)$, by taking the second derivative of the product $E \sigma_{\text {fus }}(E)$ with respect to the center-of-mass energy $E$, that is, $D^{\text {fus }}=d^{2}\left(E \sigma_{\text {fus }}\right) / d E^{2}$. This method has stimulated many high precision measurements of fusion excitation function for medium-heavy mass systems [2, 7]. The extracted barrier distributions have revealed that the concept indeed holds and the barrier distribution itself provides a powerful tool for investigating the effects of channel coupling on heavy-ion fusion reactions at sub-barrier energies. It has also been shown recently that the concept of barrier distribution is still valid even for relatively heavy systems, such as ${ }^{100} \mathrm{Mo}+{ }^{100} \mathrm{Mo}$ [8].

A similar barrier distribution can also be extracted from quasi-elastic scattering (a sum of elastic, inelastic and transfer processes) at backward angles [9, 10], that is a good counterpart of the fusion reaction [11]. In this case, the barrier distribution is defined as the first derivative of the ratio of quasi-elastic to the Rutherford cross sections $d \sigma_{\mathrm{qel}} / d \sigma_{R}$, with respect to $E$, i.e., $D^{\mathrm{qel}}=-d\left(d \sigma_{\mathrm{qel}} / d \sigma_{R}\right) / d E$. Since the fusion and the quasi-elastic scattering is related to each other because of the flux conservation, a similar information can be obtained from those processes and the similarity between the two representations for barrier distribution has been shown to hold for several intermediate mass systems [9, 10, 12].

Recently, the quasi-elastic barrier distribution has been exploited to investigate the entrance channel dynamics for fusion reactions to synthesize super-heavy elements 13, 14, 15, 16]. It has been shown that the concept of barrier distribution remains valid even for such very heavy systems once the deep-inelastic cross sections are properly taken into account. As is expected, the strong channel coupling effects on the barrier distribution have been observed.

In this paper, we carry out a detailed coupled-channels analysis for large-angle quasi-elastic scattering data for ${ }^{48} \mathrm{Ti},{ }^{54} \mathrm{Cr},{ }^{56} \mathrm{Fe},{ }^{64} \mathrm{Ni}$ and ${ }^{70} \mathrm{Zn}+{ }^{208} \mathrm{~Pb}$ systems leading to super-heavy elements $Z=104,106,108,110$, and 112, respectively [15, 16]. We especially study the role of multi-phonon excitations of the target and projectile nuclei, which has been shown to play an important role in quasi-elastic scattering for the ${ }^{86} \mathrm{Kr}+{ }^{208} \mathrm{~Pb}$ system [14].

The paper is organized as follows. We briefly explain the coupled-channels formalism for quasi-elastic scatter- 
ing in Sec. II. We present the results of our systematic analysis in Sec. III. We then summarize the paper in Sec. IV.

\section{COUPLED-CHANNELS FORMALISM FOR LARGE ANGLE QUASI-ELASTIC SCATTERING}

In this section, we briefly describe the coupled-channels formalism for large angle quasi-elastic scattering which includes the effects of the vibrational excitations of the colliding nuclei. The total Hamiltonian of the system is assumed to be

$$
\begin{aligned}
H= & -\frac{\hbar^{2}}{2 \mu} \nabla^{2}+V_{N}^{(0)}(r)+\frac{Z_{P} Z_{T} e^{2}}{r} \\
& +H_{\text {exct }}+V_{\text {coup }}\left(\boldsymbol{r}, \xi_{P}, \xi_{T}\right)
\end{aligned}
$$

where $\boldsymbol{r}$ is the coordinate of the relative motion between the target and the projectile nuclei, $\mu$ is the reduced mass and $\xi_{T}$ and $\xi_{P}$ represent the coordinate of the vibration in the target and the projectile nuclei, respectively. $Z_{P}$ and $Z_{T}$ are the atomic number of the projectile and the target, respectively, and $V_{N}^{(0)}$ is the bare nuclear potential, which we assume to have a WoodsSaxon shape. It consists of the real and imaginary parts, $V_{N}^{(0)}(r)=V_{0}(r)+i W_{0}(r) . \quad H_{\text {exct }}$ describes the excitation spectra of the target and projectile nuclei, while $V_{\text {coup }}\left(\boldsymbol{r}, \xi_{P}, \xi_{T}\right)$ is the potential for the coupling between the relative motion and the vibrational motions of the target and projectile nuclei.

In the iso-centrifugal approximation [1, 17, 18], where the the angular momentum of the relative motion in each channel is replaced with the total angular momentum $J$ (in the literature, this approximation is also referred to as the rotating frame approximation or the no-Coriolis approximation), the coupled-channels equations derived from the Hamiltonian (11) read

$$
\begin{aligned}
& {\left[-\frac{\hbar^{2}}{2 \mu} \frac{d^{2}}{d r^{2}}+\frac{J(J+1) \hbar^{2}}{2 \mu r^{2}}+V_{N}^{(0)}(r)+\frac{Z_{P} Z_{T} e^{2}}{r}\right.} \\
& \left.-E+\epsilon_{n}\right] u_{n}(r)+\sum_{n^{\prime}} V_{n n^{\prime}}(r) u_{n^{\prime}}(r)=0
\end{aligned}
$$

where $\epsilon_{n}$ is the eigen-value of the operator $H_{\text {exct }}$ for the $n$-th channel. $V_{n n^{\prime}}(r)$ is the matrix elements for the coupling potential $V_{\text {coup }}$.

In the calculations presented below, we use the method of the computer code CCFULL [17] and replace the vibrational coordinates $\xi_{P}$ and $\xi_{T}$ in the coupling potential $V_{\text {coup }}$ with the dynamical excitation operators $\hat{O}_{P}$ and $\hat{O}_{T}$. The coupling potential is then represented as

$$
V_{\text {coup }}\left(r, \hat{O}_{P}, \hat{O}_{T}\right)=V_{C}\left(r, \hat{O}_{P}, \hat{O}_{T}\right)+V_{N}\left(r, \hat{O}_{P}, \hat{O}_{T}\right)
$$

$$
\begin{aligned}
V_{C}\left(r, \hat{O}_{P}, \hat{O}_{T}\right)= & \left(\frac{3 R_{P}^{\lambda_{P}} \hat{O}_{P}}{\left(2 \lambda_{P}+1\right) r_{P}^{\lambda_{P}}}+\frac{3 R_{T}^{\lambda_{T}} \hat{O}_{T}}{\left(2 \lambda_{T}+1\right) r^{\lambda_{T}}}\right) \\
& \times \frac{Z_{P} Z_{T} e^{2}}{r}, \\
V_{N}\left(r, \hat{O}_{P}, \hat{O}_{T}\right)= & \frac{-V_{0}}{\left[1+\exp \left(\frac{\left[r-R_{0}-\left(R_{P} \hat{O}_{P}+R_{T} \hat{O}_{T}\right)\right]}{a}\right)\right]} \\
& -V_{N}^{(0)}(r) .
\end{aligned}
$$

Here, $\lambda_{P}$ and $\lambda_{T}$ denote the multipolarity of the vibrations in the projectile and the target nuclei, respectively. We have subtracted $V_{N}^{(0)}(r)$ in Eq. (5) in order to avoid the double counting.

If we truncate the phonon space up to the triple phonon states (that is, $n=0,1,2$, and 3 ), the matrix elements of the excitation operator $\hat{O}$ in Eqs. (44) and (5) are given by

$$
O_{n n^{\prime}}=\frac{1}{\sqrt{4 \pi}}\left[\begin{array}{cccc}
0 & \beta & 0 & 0 \\
\beta & 0 & \sqrt{2} \beta & 0 \\
0 & \sqrt{2} \beta & 0 & \sqrt{3} \beta \\
0 & 0 & \sqrt{3} \beta & 0
\end{array}\right]
$$

where $\beta$ is the deformation parameter, that can be estimated from a measured electric transition probability from the single phonon state $(n=1)$ to the ground state $(n=0)$. We have assumed the harmonic oscillator model for the vibrations, where $\epsilon_{n}$ in Eq. (2) is given by $\epsilon_{n}=n \hbar \omega$.

The coupled-channels equations, Eq. (2), are solved with the scattering boundary condition for $u_{n}(r)$,

$$
u_{n}(r) \rightarrow \frac{i}{2}\left\{\begin{array}{l}
\left.H_{J}^{(-)}\left(k_{n} r\right) \delta_{n, n_{i}}-\sqrt{\frac{k_{i}}{k_{n}}} S_{n}^{J} H_{J}^{(+)}\left(k_{n} r\right)\right\} \\
(r \rightarrow \infty)
\end{array}\right.
$$

where $S_{n}^{J}$ is the nuclear $S$ matrix. $H_{J}^{(-)}(k r)$ and $H_{J}^{(-)}(k r)$ are the incoming and the outgoing Coulomb wave functions, respectively. The channel wave number $k_{n}$ is given by $\sqrt{2 \mu\left(E-\epsilon_{n}\right) / \hbar^{2}}$, and $k_{i}=k_{n_{i}}=$ $\sqrt{2 \mu E / \hbar^{2}}$. The scattering angular distribution for the channel $n$ is then given by [18]

$$
\frac{d \sigma_{n}}{d \Omega}=\frac{k_{n}}{k_{i}}\left|f_{n}(\theta)\right|^{2}
$$

with

$$
\begin{array}{r}
f_{n}(\theta)=\sum_{J} e^{\left[\sigma_{J}(E)+\sigma_{J}\left(E-\epsilon_{n}\right)\right]} \sqrt{\frac{2 J+1}{4 \pi}} Y_{J 0}(\theta) \\
\times \frac{-2 i \pi}{\sqrt{k_{i} k_{n}}}\left(S_{n}^{J}-\delta_{n, n_{i}}\right)+f_{C}(\theta) \delta_{n, n_{i}}
\end{array}
$$

where $\sigma_{J}(E)$ and $f_{C}(\theta)$ are the the Coulomb phase shift and the Coulomb scattering amplitude, respectively. The 
differential quasi-elastic cross section is then calculated to be

$$
\frac{d \sigma^{\mathrm{qel}}}{d \Omega}=\sum_{n} \frac{d \sigma_{n}}{d \Omega}
$$

We will apply this formalism in the next section to analyze the quasi-elastic scattering data of ${ }^{48} \mathrm{Ti},{ }^{54} \mathrm{Cr},{ }^{56} \mathrm{Fe},{ }^{64} \mathrm{Ni}$, and ${ }^{70} \mathrm{Zn}+{ }^{208} \mathrm{~Pb}$ systems.

\section{COMPARISON WITH EXPERIMENTAL DATA : EFFECTS OF MULTI-PHONON EXCITATIONS}

In this section, we present the results of our detailed coupled-channels analysis for quasi-elastic scattering data of ${ }^{48} \mathrm{Ti},{ }^{54} \mathrm{Cr},{ }^{56} \mathrm{Fe},{ }^{64} \mathrm{Ni}$, and ${ }^{70} \mathrm{Zn}+{ }^{208} \mathrm{~Pb}$ systems [15, 16]. The calculations are performed with a version [19] of the coupled-channels code CCFULL 17]. Notice that the iso-centrifugal approximation employed in this code works well for quasi-elastic scattering at backward angles [10]. In the code, the regular boundary condition is imposed at the origin instead of the incoming wave boundary condition. We discuss the stability of the numerical calculations for the massive systems in Appendix A.

The surface diffuseness of the real part of the nuclear potential is taken to be $a=0.63 \mathrm{fm}$, as suggested by recent studies on deep sub-barrier quasi-elastic and Mott scattering 20, 21, 22], while the radius parameter to be $r_{0}=1.22 \mathrm{fm}$ for all the systems. Notice that a similar value for $a$ has been used also in the analysis of the recent experimental data for quasi-elastic scattering in the ${ }^{86} \mathrm{Kr}+{ }^{208} \mathrm{~Pb}$ system [14]. The depth parameter, $V_{0}$, is adjusted in order to reproduce the experimental quasi-elastic cross sections for each system. The optimum values of the depth parameter and the resultant Coulomb barrier height are summarized in Table I. As usually done, we use a short range imaginary potential with $W_{0}=30 \mathrm{MeV}, r_{w}=1.0 \mathrm{fm}$ and $a_{w}=0.3 \mathrm{fm}$ to simulate the compound nucleus formation. The results are insensitive to these parameters as long as the imaginary part of the potential is well confined inside the Coulomb barrier. The excitation energy and the corresponding deformation parameter for the single phonon excitation in each nucleus included in the calculations are given in Table II. The latter quantity is taken from Refs. [23, 24]. The radius of the target and the projectile are taken to be $R_{T}=1.2 A_{T}^{1 / 3}$ and $R_{P}=1.2 A_{P}^{1 / 3}$, respectively, in order to be consistent with the deformation parameters [23, 24]. All the calculations shown below are performed at the scattering angle of $\theta_{\text {c.m. }}=170^{\circ}$. We plot the quasi-elastic cross sections and barrier distributions as a function of the effective energy defined by $[9,10]$

$$
E_{\text {eff }}=2 E \frac{\sin (\theta / 2)}{1+\sin (\theta / 2)},
$$

TABLE I: The depth parameter for the real part of the nuclear potential for the ${ }^{48} \mathrm{Ti},{ }^{54} \mathrm{Cr},{ }^{56} \mathrm{Fe},{ }^{64} \mathrm{Ni}$, and ${ }^{70} \mathrm{Zn}+{ }^{208} \mathrm{~Pb}$ systems. The radius and the diffuseness parameters are taken to be $r_{0}=1.22 \mathrm{fm}$ and $a=0.63 \mathrm{fm}$, respectively, for all the systems. The resultant barrier height energy $V_{B}$ is also listed.

\begin{tabular}{ccc}
\hline \hline System & $V_{0}(\mathrm{MeV})$ & $V_{B}(\mathrm{MeV})$ \\
\hline${ }^{48} \mathrm{Ti}+{ }^{208} \mathrm{~Pb}$ & 88.90 & 190.50 \\
${ }^{54} \mathrm{Cr}+{ }^{208} \mathrm{~Pb}$ & 91.70 & 205.50 \\
${ }^{56} \mathrm{Fe}+{ }^{208} \mathrm{~Pb}$ & 92.85 & 222.50 \\
${ }^{64} \mathrm{Ni}+{ }^{208} \mathrm{~Pb}$ & 95.10 & 236.25 \\
${ }^{70} \mathrm{Zn}+{ }^{208} \mathrm{~Pb}$ & 108.2 & 249.30 \\
\hline \hline
\end{tabular}

TABLE II: The properties of the single phonon states included in the present coupled-channels calculations. $\hbar \omega$ and $\beta$ are the excitation energy and the dynamical deformation parameter, respectively.

\begin{tabular}{clcc}
\hline \hline Nucleus & $I^{\pi}$ & $\hbar \omega(\mathrm{MeV})$ & $\beta$ \\
\hline${ }^{208} \mathrm{~Pb}$ & $3^{-}$ & 2.614 & $0.110^{a}$ \\
${ }^{48} \mathrm{Ti}$ & $2^{+}$ & 0.983 & $0.269^{b}$ \\
${ }^{54} \mathrm{Cr}$ & $2^{+}$ & 0.834 & $0.250^{b}$ \\
${ }^{56} \mathrm{Fe}$ & $2^{+}$ & 0.846 & $0.239^{b}$ \\
${ }^{64} \mathrm{Ni}$ & $2^{+}$ & 1.346 & $0.179^{b}$ \\
${ }^{70} \mathrm{Zn}$ & $2^{+}$ & 0.884 & $0.228^{b}$ \\
\hline \hline
\end{tabular}

${ }^{a}$ taken from Ref. 23.

${ }^{b}$ taken from Ref. [24].

which takes into account the centrifugal energy. We calculate the quasi-elastic barrier distributions from the cross sections in a similar way as the one used to obtain the experimental barrier distributions [16]. Namely, we use the point difference formula with the energy step of $\Delta E=0.25 \mathrm{MeV}$ and then smooth the resultant barrier distribution with the Gaussian function with the full width at half maximum (FWHM) of $1.5 \mathrm{MeV}$. We have checked that the shape of the barrier distribution does not change significantly even if we use a larger energy step for the point difference formula, e.g. $\Delta E=0.5 \mathrm{MeV}$.

\section{A. Effect of double phonon excitations}

Let us first discuss the effect of double octupole phonon excitations in the ${ }^{208} \mathrm{~Pb}$ target. Such excitations have been shown to play a significant role in the sub-barrier fusion reaction between ${ }^{16} \mathrm{O}$ and ${ }^{208} \mathrm{~Pb}$ nuclei [25, 26].

The dotted line in Fig. 1 shows the result of the coupled-channels calculation for the ${ }^{48} \mathrm{Ti}+{ }^{208} \mathrm{~Pb}$ system obtained by taking into account the coupling to the single octupole phonon state in the target nucleus, ${ }^{208} \mathrm{~Pb}$, and the single quadrupole phonon state in the projectile nucleus, ${ }^{48} \mathrm{Ti}$. The mutual excitations in the projectile and the target nuclei are fully taken into account in this calculation as well as in all the other calculations presented in this paper. Figs. 廿(a) and 廿(b) show the ratio of the quasi-elastic to the Rutherford cross sections, $d \sigma_{\mathrm{qel}} / d \sigma_{R}$, 


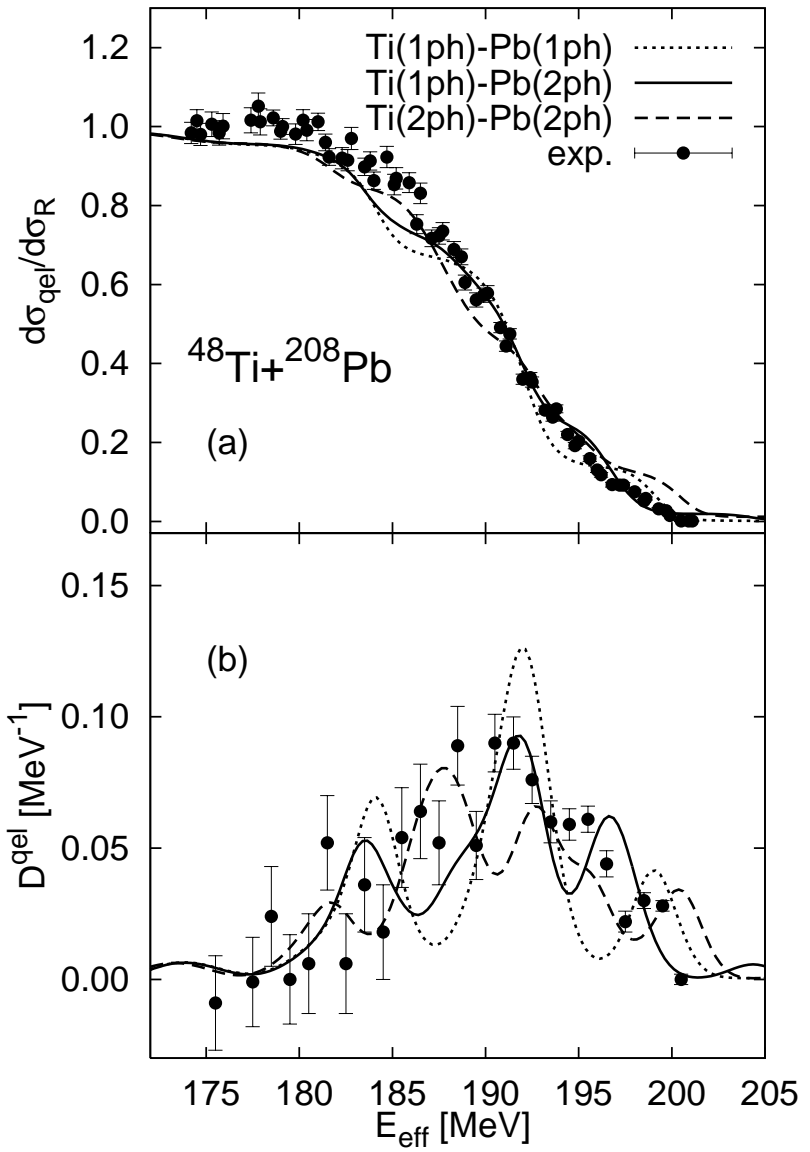

FIG. 1: Effects of multi-phonon excitations on the quasielastic scattering cross section (upper panel) and on the quasielastic barrier distribution (lower panel) for the ${ }^{48} \mathrm{Ti}+{ }^{208} \mathrm{~Pb}$ system. The dotted line is the result of the coupled-channels calculations including coupling to the one quadrupole phonon state in the projectile and the one octupole phonon states in the target nucleus, while the solid line is obtained by including the coupling in addition to the two octupole phonon state in the target nucleus. The dashed line is the result of double quadrupole phonon couplings in the projectile and the double octupole phonon couplings in the target nucleus. The experimental data are taken from Ref. [16].

and the quasi-elastic barrier distribution, $D^{\mathrm{qel}}$, respectively. Although the overall width of the barrier distribution is reproduced reasonably well with this calculation, the detailed structure is somewhat inconsistent with the experimental data. The situation is similar even when we include the double quadrupole phonon state in the projectile while keeping the single octupole phonon coupling in the target nucleus (not shown). We then investigate the effect of the double octupole phonon couplings in the target nucleus. The solid and the dashed lines in Fig. 1 show the results with the single and the double quadrupole phonon excitations in the projectile, respectively, where as the double octupole phonon coupling in the target is included in both the calculations. The former calculation reproduces both the cross sections
TABLE III: The value of $\chi^{2}$ for the quasi-elastic cross sections for the ${ }^{48} \mathrm{Ti}+{ }^{208} \mathrm{~Pb}$ system obtained with the coupledchannels calculations with various coupling schemes. The coupling schemes are denoted as $\left[n_{2}, n_{3}\right]$, where $n_{2}$ is the number of quadrupole phonon excitation in the projectile nucleus while $n_{3}$ the number of octupole phonon in the target nucleus.

\begin{tabular}{ccccc}
\hline \hline System & {$[1,1]$} & {$[1,2]$} & {$[1,3]$} & {$[2,2]$} \\
\hline${ }^{48} \mathrm{Ti}+{ }^{208} \mathrm{~Pb}$ & 19.15 & 9.82 & 7.12 & 37.12 \\
\hline \hline
\end{tabular}

and the barrier distribution reasonably well, although the latter calculation somehow worsens the agreement. This clearly suggests that the double octupole phonon excitations in the target nucleus is important in the quasielastic ${ }^{48} \mathrm{Ti}+{ }^{208} \mathrm{~Pb}$ scattering. We summarize the $\chi^{2}$ value of our calculations in Table III.

Since the coupling to the one quadrupole phonon state in the projectile and the two octupole phonon states in the target reasonably well reproduce the experimental quasi-elastic scattering data for the ${ }^{48} \mathrm{Ti}+{ }^{208} \mathrm{~Pb}$ system, one may expect that the same coupling scheme accounts for the experimental data for the other systems, ${ }^{54} \mathrm{Cr},{ }^{56} \mathrm{Fe},{ }^{64} \mathrm{Ni}$ and ${ }^{70} \mathrm{Zn}+{ }^{208} \mathrm{~Pb}$. The results of the coupled-channels calculations with this coupling scheme is shown by the dashed line in Fig. 2. Figs. 2(a), (c), (e), and $(\mathrm{g})$ are for the quasi-elastic cross sections for the ${ }^{54} \mathrm{Cr},{ }^{56} \mathrm{Fe},{ }^{64} \mathrm{Ni}$ and ${ }^{70} \mathrm{Zn}+{ }^{208} \mathrm{~Pb}$ systems, respectively, while Figs. 2(b), (d), (f), and (h) are for the quasi-elastic barrier distributions. One can clearly see that these calculations underestimate the experimental cross sections at high energies, although the experimental barrier distributions themselves are reproduced reasonably well. We repeat the same calculations by including the coupling up to the double quadrupole phonon states in the projectile, in addition to the double octupole phonon states in the target nucleus. These results are shown by the solid in Fig. 2, The agreement with the experimental data is considerably improved, especially for the quasielastic cross sections. See Table IV for the $\chi^{2}$ values. It is thus evident that the coupling to the double quadrupole phonon states in the projectile is needed in order to explain the experimental data for the ${ }^{54} \mathrm{Cr},{ }^{56} \mathrm{Fe},{ }^{64} \mathrm{Ni}$ and ${ }^{70} \mathrm{Zn}+{ }^{208} \mathrm{~Pb}$ reactions.

The reason why the double quadrupole phonon coupling is not necessary for the ${ }^{48} \mathrm{Ti}$ projectile while it is for the heavier projectiles is not clear at this moment. This might reflect some ambiguity of the Monte Carlo reaction simulation code LINDA [27] which was used to subtract the deep-inelastic component from the experimental yields at backward angles [16]. Clearly, a further investigation is still necessary concerning the effect of deep inelastic scattering on quasi-elastic scattering in massive systems [13, 14, 15, 16]. 

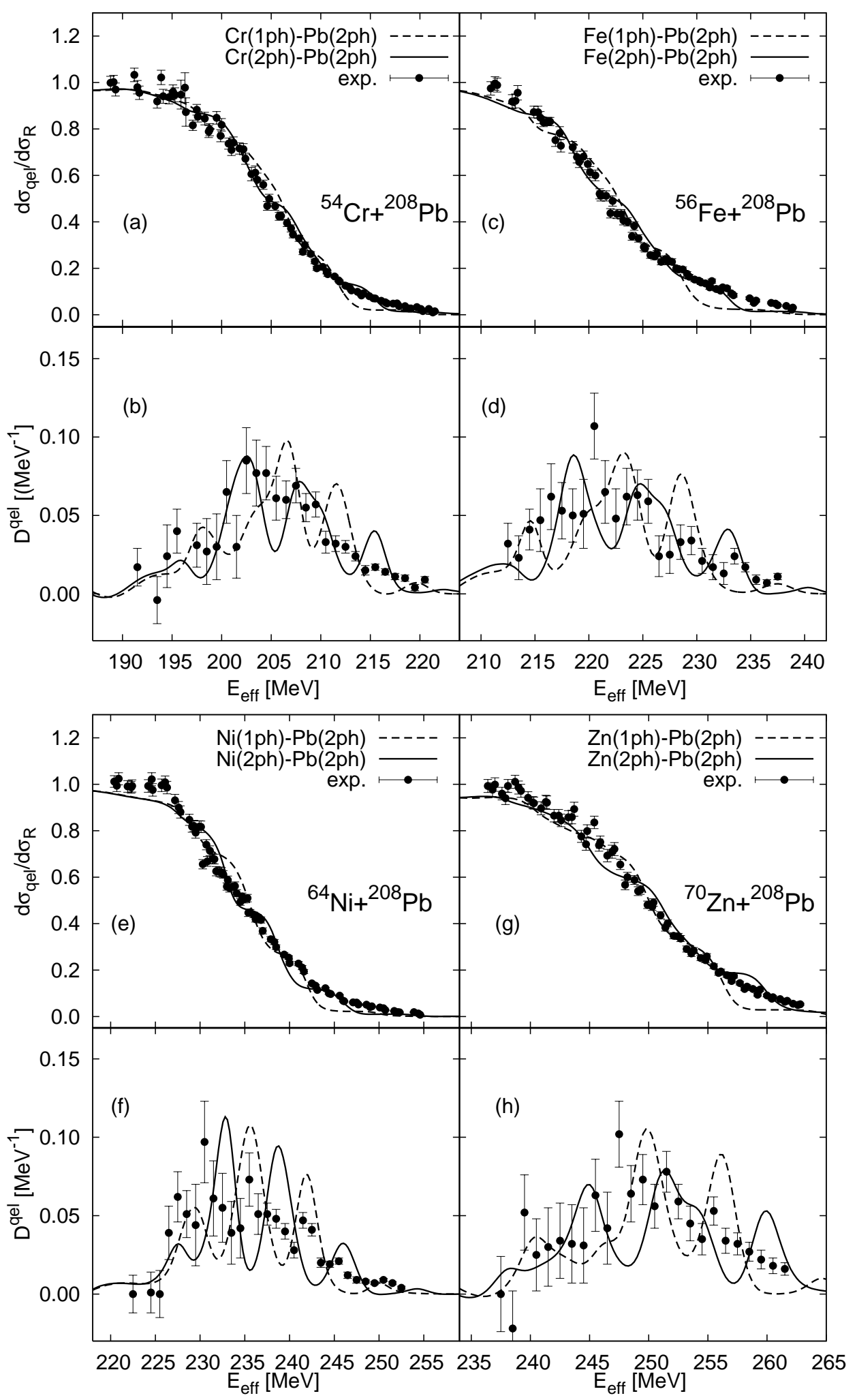

FIG. 2: The quasi-elastic scattering cross sections ((a), (c), (e), and (g)) and the quasi-elastic barrier distributions ((b), (d), $(\mathrm{f})$, and $(\mathrm{h})$ ) for the ${ }^{54} \mathrm{Cr},{ }^{56} \mathrm{Fe},{ }^{64} \mathrm{Ni}$ and ${ }^{70} \mathrm{Zn}+{ }^{208} \mathrm{~Pb}$ systems obtained with two coupling schemes as indicated in the insets. The dashed line is obtained by including the one quadrupole phonon state in the projectile nuclei while the solid line is obtained with the double phonon couplings. The double octupole phonon excitations in the target nucleus is included in all the calculations. The experimental data are taken from Ref. [16]. 


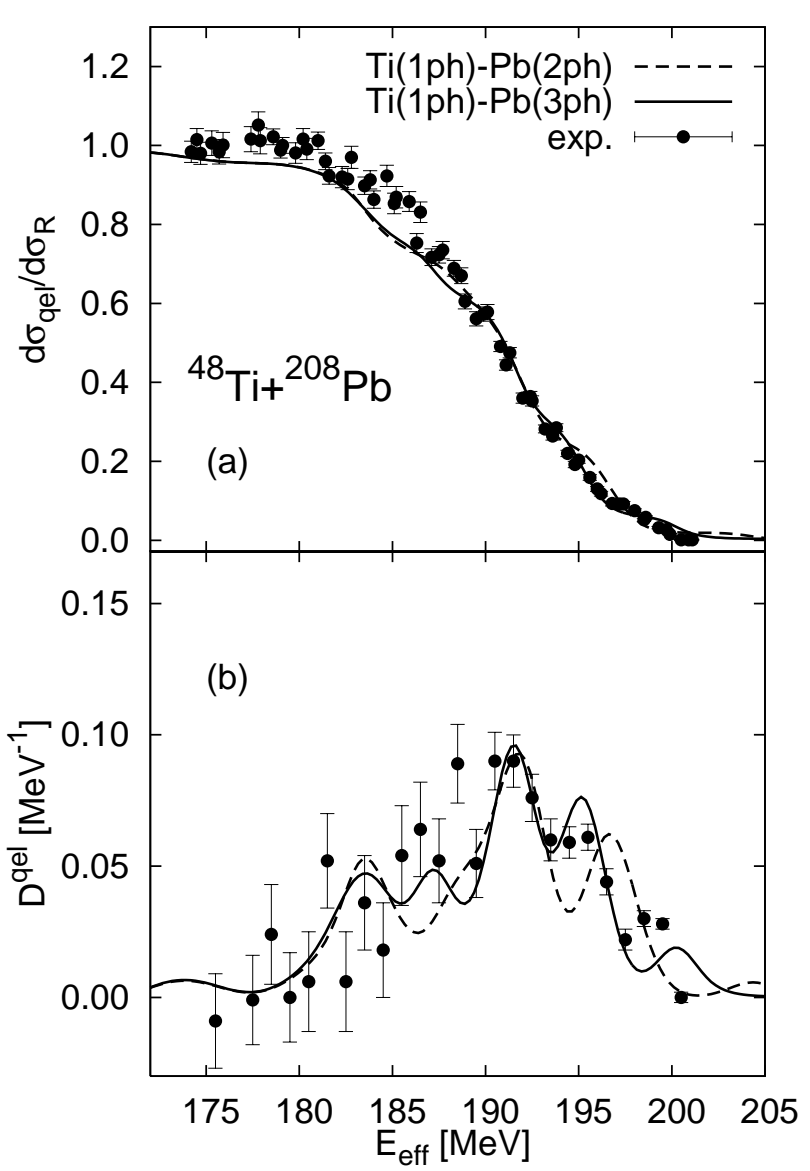

FIG. 3: Effects of triple phonon excitations on the quasielastic scattering cross section (upper panel) and on the quasielastic barrier distribution (lower panel) for the ${ }^{48} \mathrm{Ti}+{ }^{208} \mathrm{~Pb}$ system. The dashed line is the result of the coupled-channels calculations taking into account the coupling to the one phonon state in the projectile and the two phonon states in the target nuclei. The solid line is obtained by including the coupling to the one phonon state in the projectile and the three phonon states in the target. The experimental data are taken from Ref. [16].

TABLE IV: Same as Table III but for the ${ }^{54} \mathrm{Cr},{ }^{56} \mathrm{Fe},{ }^{64} \mathrm{Ni}$ and ${ }^{70} \mathrm{Zn}+{ }^{208} \mathrm{~Pb}$ systems.

\begin{tabular}{ccccc}
\hline \hline System & {$[1,2]$} & {$[1,3]$} & {$[2,2]$} & {$[2,3]$} \\
\hline${ }^{54} \mathrm{Cr}+{ }^{208} \mathrm{~Pb}$ & 52.47 & 49.80 & 20.61 & 11.78 \\
${ }^{56} \mathrm{Fe}+{ }^{208} \mathrm{~Pb}$ & 28.46 & 28.36 & 10.44 & 10.28 \\
${ }^{64} \mathrm{Ni}+{ }^{208} \mathrm{~Pb}$ & 57.45 & 61.43 & 32.21 & 30.64 \\
${ }^{70} \mathrm{Zn}+{ }^{208} \mathrm{~Pb}$ & 26.52 & 24.81 & 11.36 & 6.87 \\
\hline \hline
\end{tabular}

\section{B. Effect of triple phonon excitations}

In the previous subsection, we have shown that the double octupole phonon excitations in the ${ }^{208} \mathrm{~Pb}$ target play an important role in quasi-elastic scattering for the systems considered in this paper. However, the calculated quasi-elastic barrier distributions have a much more prominent peak than the experimental distribution at high energies. Since it has been shown in Refs. 13, 14 that the triple octupole phonon excitations of the ${ }^{208} \mathrm{~Pb}$ play a significant role in the large-angle quasi-elastic scattering between ${ }^{86} \mathrm{Kr}$ and ${ }^{208} \mathrm{~Pb}$ nuclei, it is intriguing to investigate such effects in the present systems as well.

The results of the coupled-channels calculations including the coupling to the triple octupole phonon states in ${ }^{208} \mathrm{~Pb}$ for the ${ }^{48} \mathrm{Ti}+{ }^{208} \mathrm{~Pb}$ reaction is presented in Fig. 3 . The dashed line is the same as the solid line in Fig. 1, that is the result of single phonon in ${ }^{48} \mathrm{Ti}$ and double phonon in ${ }^{208} \mathrm{~Pb}$. The solid line denotes the results of the triple phonon coupling in the target in addition to the single phonon in the projectile. By including the triple octupole phonons in the target nucleus, the quasi-elastic cross sections are improved slightly (see also Table III). On the other hand, one can see that the agreement for the barrier distribution with the experimental data is much more improved by the triple phonon coupling.

The results for the other systems, ${ }^{54} \mathrm{Cr},{ }^{56} \mathrm{Fe},{ }^{64} \mathrm{Ni}$ and ${ }^{70} \mathrm{Zn}+{ }^{208} \mathrm{~Pb}$ reactions, are shown in Fig. 4. Figs. 4(a), (c), (e) and (g) are for the quasi-elastic cross sections, while Figs. 4(b), (d), (f) and (h) for the quasi-elastic barrier distributions. Let us first discuss the calculations with the single phonon excitation in the projectile. The dotted line in the figures is obtained by taking the coupling to the single phonon state in the projectile and the triple octupole phonon excitations in the target. This calculation underestimates the quasi-elastic cross sections at high energies and the obtained barrier distribution is inconsistent with the experimental data. Therefore, the previous results shown in Fig. 2 is not improved even if the triple phonon excitations in the target is taken into account as long as only the single phonon excitation is considered for the projectile nucleus. The results with the double phonon couplings in the projectile together with the triple phonon excitations in the target are then shown by the solid line in the figure. For comparison, we also show by the dashed line the results of the double phonon excitations in both the projectile and the target nuclei, which is the same as the solid line in Fig. 2, One can observe that the inclusion of the triple octupole phonon excitations in the ${ }^{208} \mathrm{~Pb}$ somewhat improves the agreement between the calculations and the experimental data for both the quasi-elastic cross sections and the barrier distributions (see also Table IV).

In Ref. 14], Ntshangase et al. reduced the coupling strength of $\left(3^{-}\right) \rightarrow\left(3^{-}\right)^{2}$ states in ${ }^{208} \mathrm{~Pb}$ by a factor of $(0.6)$ and that of $\left(3^{-}\right)^{2} \rightarrow\left(3^{-}\right)^{3}$ by $(0.6)^{2}$ in order to explain the experimental barrier distribution for the ${ }^{86} \mathrm{Kr}+{ }^{208} \mathrm{~Pb}$ reaction. In order to see whether such reduction of the coupling strengths improves the agreement between the coupled-channels calculations and the experimental data for the present systems, we repeat the calculations by including those effects for the ${ }^{70} \mathrm{Zn}+{ }^{208} \mathrm{~Pb}$ system. The results are shown in Fig. 5. The solid is obtained by reducing the coupling strengths as Ntshangase et al. did, while the dashed line is the same as the solid 

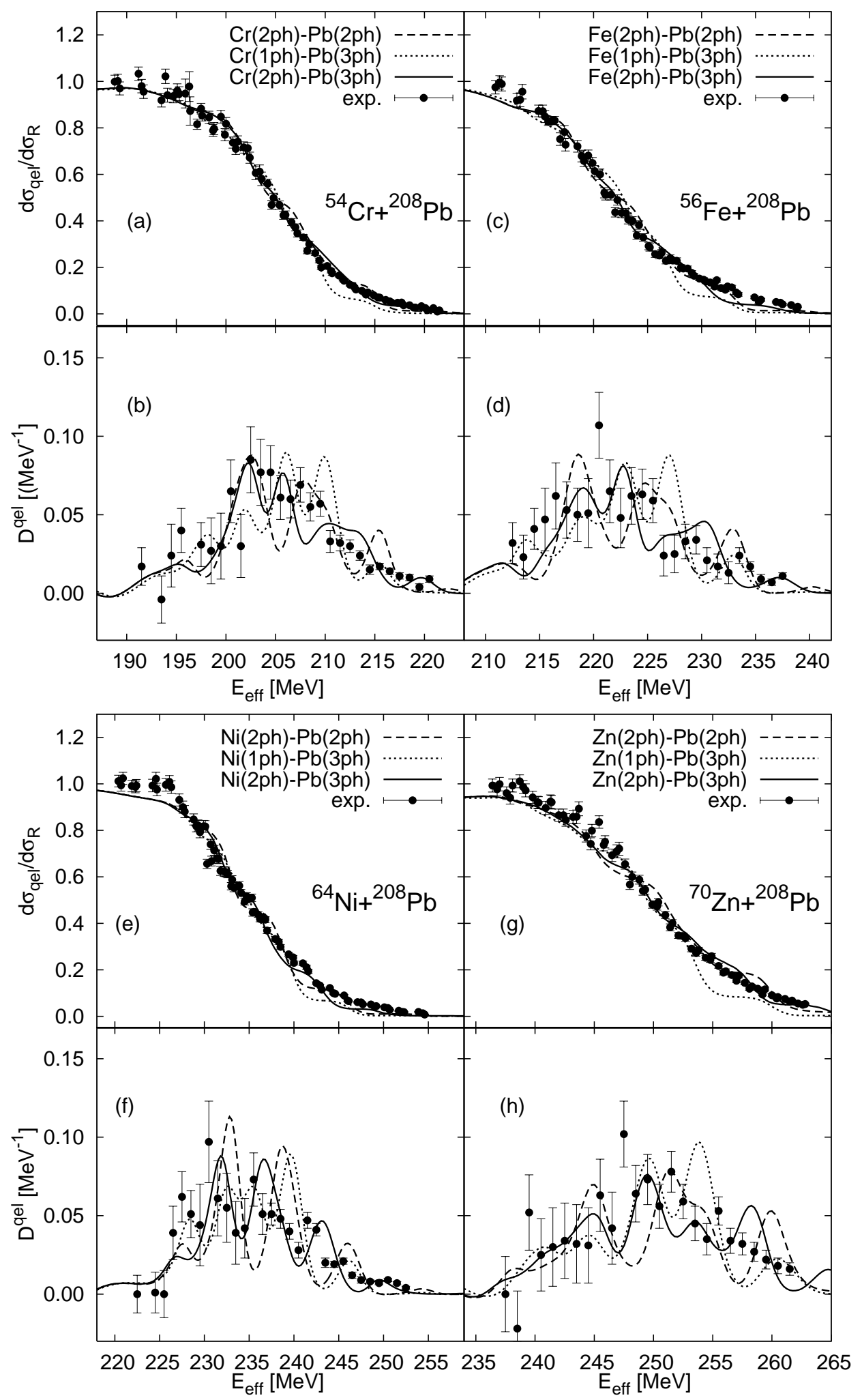

FIG. 4: Effects of triple phonon excitations on the quasi-elastic cross sections ((a), (c), (e), and (g)) and on the quasi-elastic barrier distributions ((b), (d), (f), and (h)) for the ${ }^{54} \mathrm{Cr},{ }^{56} \mathrm{Fe},{ }^{64} \mathrm{Ni}$ and ${ }^{70} \mathrm{Zn}+{ }^{208} \mathrm{~Pb}$ systems. The dashed line is the same as the solid line in Fig. 2 while the dotted line is the results of the calculations taking the coupling to the triple octupole phonon in the target and the one quadrupole phonon state in the projectile nucleus into account. The solid line is obtained by including the coupling to the double quadrupole phonon states in the projectile and the triple otcupole phonon states in the target nucleus. The experimental data are taken from Ref. [16]. 


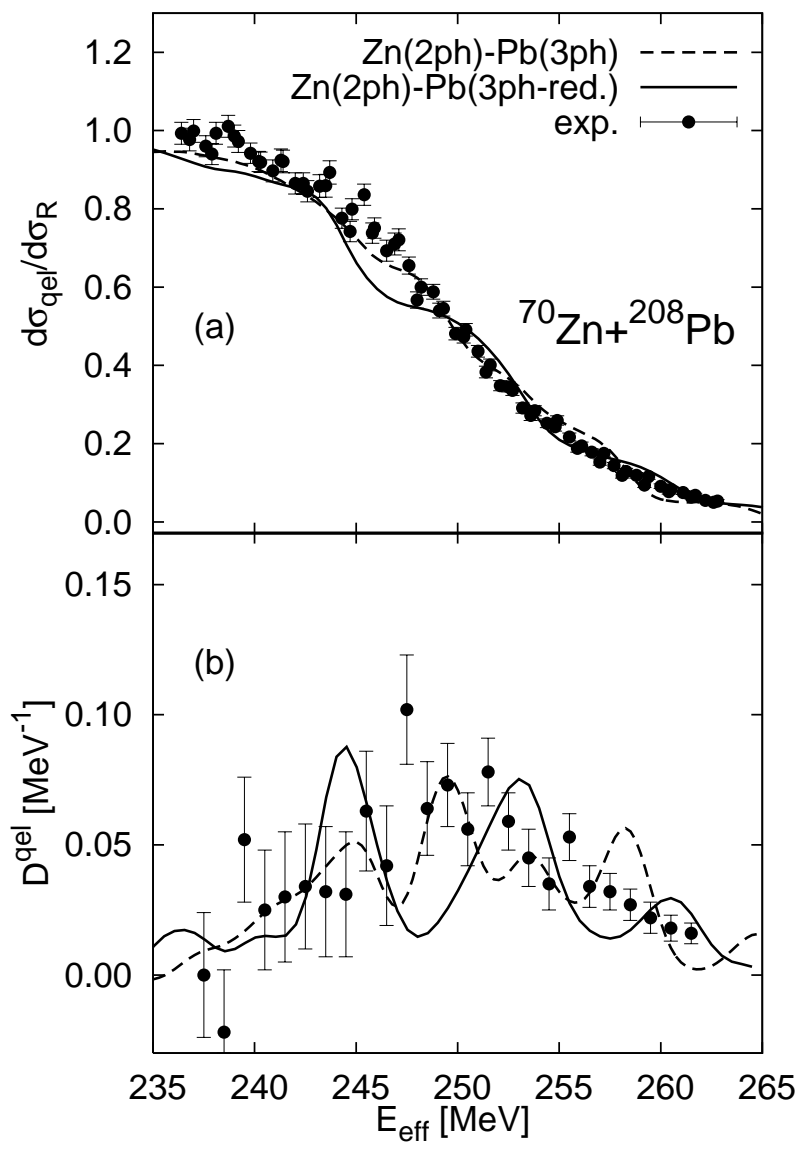

FIG. 5: Effect of anharmonic octupole phonon excitations in ${ }^{208} \mathrm{~Pb}$ on (a) the quasi-elastic cross section and (b) the quasi-elastic barrier distribution for the ${ }^{70} \mathrm{Zn}+{ }^{208} \mathrm{~Pb}$ reaction. The solid line is the coupled-channels calculation obtained by reducing the coupling strengths to multi-phonon states, while the dashed line denotes the results in the harmonic limit. The experimental data are taken from Ref. [16].

line in Figs. $4(\mathrm{~g})$ and $(\mathrm{h})$, that is obtained by assuming the harmonic limit. In both cases, we take into account the coupling to the double quadrupole phonon excitations in the projectile nucleus. One can see that the harmonic model leads to a better agreement with the experimental data both for the cross sections and the barrier distribution, as compared to the anharmonic calculation. The difference between Ref. [14] and the present calculation concerning the role of anharmonicity may originate from the fact that Ref. [14] used a smaller value for $R_{T}$ $\left(=1.06 A_{T}^{1 / 3} \mathrm{fm}\right)$ and thus a larger value for $\beta_{3}(=0.16)$. In order to clarify the role of anharmonicity of multi-phonon excitations in quasi-elastic scattering in massive systems, it would be required to take into account also the reorientation terms [12, 28, 29]. It is beyond the scope of this paper, and we will leave it for a future study.

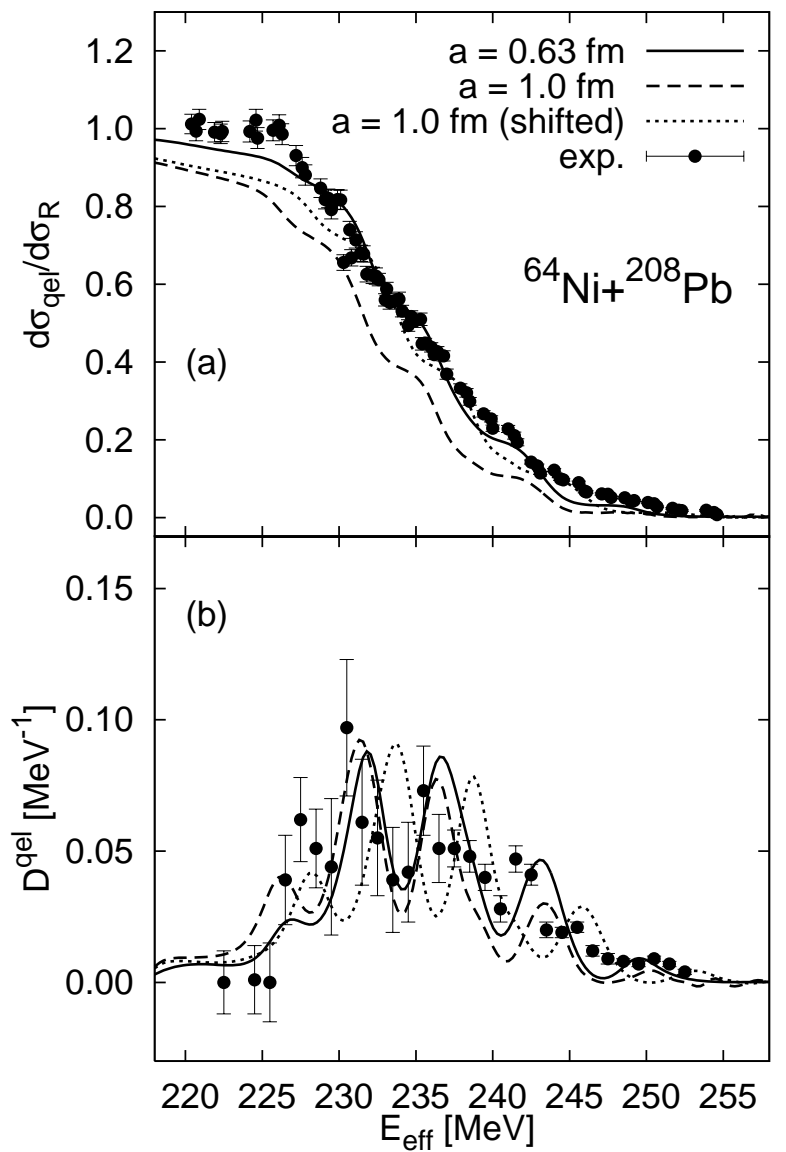

FIG. 6: Comparison of the experimental data with the coupled-channels calculations obtained using different values of the surface diffuseness of the nuclear potential for ${ }^{64} \mathrm{Ni}+{ }^{208} \mathrm{~Pb}$ reaction for (a) the quasi-elastic cross section and (b) the quasi-elastic barrier distribution. The solid and the dashed lines is obtained using the surface diffuseness of the nuclear potential $a=0.63 \mathrm{fm}$ and $a=1.0 \mathrm{fm}$, respectively. The dotted line is the results obtained by shifting the barrier height by around $+2.40 \mathrm{MeV}$ for the calculation using $a=1.0$ fm. Experimental data is taken from Ref. [16].

\section{Surface diffuseness of the nuclear potential}

We next discuss the dependence of the quasi-elastic scattering on the surface diffuseness parameter of the nuclear potential. The standard value for the diffuseness parameter is around $0.63 \mathrm{fm}[30,31,32]$. Recently, systematic studies on quasi-elastic scattering as well as Mott scattering at deep sub-barrier energies have revealed that the surface region of the nuclear potential is indeed consistent with the standard value of the surface diffuseness parameter [20, 21, 22]. On the other hand, it has been known for some time that the recent high precision data of sub-barrier fusion cross sections require a larger value of surface diffuseness parameter, ranging between 0.75 and $1.5 \mathrm{fm} \mathrm{[33]}$. Since the large-angle quasi-elastic scattering around the Coulomb barrier may probe both the 
surface region and the inner part of the nuclear potential, it is interesting to study the sensitivity of quasi-elastic cross sections and barrier distributions to the surface diffuseness parameter.

For this purpose, as an example, we repeat the coupledchannels calculation for the the ${ }^{64} \mathrm{Ni}+{ }^{208} \mathrm{~Pb}$ reaction using the nuclear potential with $a=1.0 \mathrm{fm}$. We readjust the depth and the radius parameters to be $V_{0}=160.70 \mathrm{MeV}$ and $r_{0}=1.10 \mathrm{fm}$, respectively, so that the barrier height remains the same as the one listed in Table I. We include the coupling to the double phonon states in the projectile and the triple phonon states in the target. Fig. 6 compares the results with $a=0.63 \mathrm{fm}$ (the solid line) to the one with $a=1.0 \mathrm{fm}$ (the dashed line). One sees that the calculations with $a=1.0 \mathrm{fm}$ underestimate the quasielastic cross section, although the shape of barrier distribution itself is similar to the one obtained with $a=0.63$ $\mathrm{fm}$. The dotted line is obtained with the same value of surface diffuseness parameter $a=1.0 \mathrm{fm}$ as the one for the dashed line, but by changing the depth parameter $V_{0}$ so that the resultant barrier height is higher by $2.4 \mathrm{MeV}$. This calculation now reproduces the experimental quasielastic cross sections at energies larger than $E_{\text {c.m. }}=230$ $\mathrm{MeV}$ reasonably well, but below this energy the cross sections are underestimated. Therefore, it seems difficult to reproduce the experimental quasi-elastic cross sections with the diffuseness parameter of $a=1.0 \mathrm{fm}$ at energies below and above the Coulomb barrier simultaneously. We have checked that the situation is similar for the other systems, ${ }^{48} \mathrm{Ti},{ }^{54} \mathrm{Cr},{ }^{56} \mathrm{Fe}$ and ${ }^{70} \mathrm{Zn}+{ }^{208} \mathrm{~Pb}$. This result clearly indicates that the standard value of surface diffuseness, $a=0.63 \mathrm{fm}$, is preferred by the experimental quasi-elastic data for the systems studied in this paper.

\section{SUMMARY}

We have performed a detailed coupled-channels analysis for large-angle quasi-elastic scattering of the ${ }^{48} \mathrm{Ti},{ }^{54} \mathrm{Cr},{ }^{56} \mathrm{Fe},{ }^{64} \mathrm{Ni}$ and ${ }^{70} \mathrm{Zn}+{ }^{208} \mathrm{~Pb}$ systems, where their experimental barrier distributions have been extracted recently. Our coupled-channels calculations with multi-phonon excitations in the colliding nuclei reproduce the experimental quasi-elastic cross sections as well as the barrier distributions, indicating clearly that the coupled-channels approach still works even for massive systems [8]. It was crucial to subtract properly the deepinelastic components from the total backward-angle cross sections in order to reach these agreements between the calculations and the experimental data.

In more details, the calculation with the single quadrupole phonon excitation in ${ }^{48} \mathrm{Ti}$ and the triple octupole phonon excitations in ${ }^{208} \mathrm{~Pb}$ reproduces reasonably well the experimental data for the ${ }^{48} \mathrm{Ti}+{ }^{208} \mathrm{~Pb}$ system. On the other hand, for the ${ }^{54} \mathrm{Cr},{ }^{56} \mathrm{Fe},{ }^{64} \mathrm{Ni}$ and ${ }^{70} \mathrm{Zn}+{ }^{208} \mathrm{~Pb}$ systems, we found that the coupling to the double quadrupole phonon excisions in the projectile nucleus in addition to the coupling to the triple octupole phonon in the target nucleus seems to be needed to fit the experimental data. These results suggest that the triple octupole phonon excitations in the ${ }^{208} \mathrm{~Pb}$ nucleus plays an important role in describing the experimental data for the quasi-elastic cross section and the quasi-elastic barrier distribution for the present massive systems. This is consistent with the previous finding for the ${ }^{86} \mathrm{Kr}+{ }^{208} \mathrm{~Pb}$ system [14].

Although our calculations well reproduce the gross features of the experimental barrier distributions, higher precision data are still required in order to study the detailed structure of the barrier distributions, especially the role of multi octupole phonon states in ${ }^{208} \mathrm{~Pb}$. From the theoretical side, a further detailed investigation will also be necessary, taking into account the anharmonicity of the multi-phonon excitations.

\section{Acknowledgments}

This work was partly supported by The 21st Century Center of Excellence Program "Exploring New Science by Bridging Particle-Matter Hierarchy" of Tohoku University and partly by Monbukagakusho Scholarship and Grant-in-Aid for Scientific Research under the program number 19740115 from the Japanese Ministry of Education, Culture, Sports, Science and Technology.

\section{APPENDIX A: NUMERICAL STABILIZATION OF COUPLED-CHANNELS CALCULATION}

In this Appendix, we discuss the problem of numerical instability of coupled-channels calculations and the stabilization methods which we employ in the present calculations.

The coupled-channels equations (2) form a set of $N$ second order coupled linear differential equations, where $N$ is the dimension of the coupled-channels equations. These equations can be solved by generating $N$ linearly independent solutions and taking a linear combination of these $N$ solutions so that the asymptotic boundary condition, (7), as well as the regular boundary condition at the origin, are satisfied. The linearly independent solutions can be obtained by taking $N$ different sets of initial conditions at $r=0$. We denote these solutions by $\phi_{n n_{i}}(r)$, where $n$ refers to the channels while $n_{i}$ refers to a particular choice of the initial conditions. A simple choice for the $N$ initial conditions is to impose

$$
\phi_{n m}(r) \rightarrow c r^{J+1} \delta_{n, m}, \quad \text { for } r \rightarrow 0,
$$

where $c$ is an arbitrary number and $J$ is the total angular momentum. With these boundary condition, the 
coupled-channels equations for $\phi_{n m}(r)$ given by

$$
\begin{aligned}
& {\left[-\frac{\hbar^{2}}{2 \mu} \frac{d^{2}}{d r^{2}}+\frac{J(J+1) \hbar^{2}}{2 \mu r^{2}}+V_{N}^{(0)}(r)+\frac{Z_{P} Z_{T} e^{2}}{r}\right.} \\
& \left.-E+\epsilon_{n}\right] \phi_{n m}(r)+\sum_{n^{\prime}} V_{n n^{\prime}}(r) \phi_{n^{\prime} m}(r)=0,
\end{aligned}
$$

are solved outwards up to a matching radius $R_{\max }$. The wave functions $u_{n}(r)$ in Eq. (2) are then obtained as

$$
u_{n}(r)=\sum_{m} C_{m} \phi_{n m}(r),
$$

where the coefficients $C_{m}$ are determined so that the asymptotic boundary condition (7) is fulfilled.

In the classical forbidden region, the scattering wave functions exponentially damp as the coordinate $r$ decreases. For the smaller energy, the damping is the stronger. Therefore, when the excitation energy $\epsilon_{n}$ is finite, the absolute value of the wave functions for each channel are different by order of magnitude in the classical forbidden region, and thus the wave functions tend to be dominated by that of the channel which has the smallest excitation energy. This easily destroys the linear independence of the $N$ numerical solutions $\phi_{n m}$, and causes the numerical instability. This is a serious problem especially when the coupling is strong, as in the massive systems which we discuss in this paper.

Several methods have been proposed in order to stabilize the numerical solution of coupled-channels equations [34, 35, 36, 37]. In the present calculations, we stabilize the solutions by diagonalizing the wave function matrix $\phi_{n m}$ at several points of $r$ in order to recover the linear independence. That is, at some radius $r_{s}$, we compute the inverse of the matrix $A_{n m}=\phi_{n m}\left(r_{s}\right)$, and define the new set of wave functions,

$$
\tilde{\phi}_{n m}(r)=\sum_{k} \phi_{n k}(r) \cdot\left(A^{-1}\right)_{k m} .
$$

The new wave functions $\tilde{\phi}$ obey similar coupled-channels equations as Eq. (A2), with the boundary conditions given by,

$$
\begin{aligned}
\tilde{\phi}_{n m}\left(r_{s}-h\right) & =\sum_{k} \phi_{n k}(r-h) \cdot\left(A^{-1}\right)_{k m}, \\
\tilde{\phi}_{n m}\left(r_{s}\right) & =\delta_{n, m} .
\end{aligned}
$$

Here, $h$ is the step for the discretization of the radial coordinate, $r$. These coupled-channels equations are solved outwards from $r_{s}$. The solutions $\phi$ can then be constructed as $\phi=A \cdot \tilde{\phi}$. We impose this stabilization procedure up to $r=15 \mathrm{fm}$ with an interval of $1 \mathrm{fm}$. Although this method is similar to those in Refs. [36, 37], our method is much simpler to be implemented.

This method is sufficient for intermediate heavy systems, such as ${ }^{16} \mathrm{O}+{ }^{144} \mathrm{Sm}$. For massive systems, however, we still encounter a small numerical instability [38]. In order to cure this problem, in addition to the stabilization method (A4), we also adopt two other methods, which are used in the computer code FRESCO [39]. That is, we introduce two radii, $R_{\min }$ and $R_{\text {cut }}$. $R_{\min }$ is the radius from which the coupled-channels equations (A2) are solved, i.e., these equations are solved from $r=R_{\min }$ instead of $r=0$, by setting $\phi_{n m}(r)=0$ for $r \leq R_{\text {min }}$. $R_{\text {cut }}$ is a cut-off radius for the coupling matrix, i.e., the offdiagonal components of the coupling matrix $V_{n n^{\prime}}(r)$ are set to be zero for $r \leq R_{\text {cut }}$. Both the procedures are justified when the absorption is strong inside the Coulomb barrier, as in heavy-ion systems, and the results are insensitive to the particular choice of $R_{\min }$ and $R_{\text {cut }}$ as long as they are inside the Coulomb barrier. Typically, we take $R_{\text {min }}=6 \mathrm{fm}$ and $R_{\text {cut }}=10 \mathrm{fm}$ to obtain reasonable results for the present systems (notice that the pocket and the barrier appear at e.g., 11.3 and $13.2 \mathrm{fm}$, respectively, for the ${ }^{64} \mathrm{Ni}+{ }^{208} \mathrm{~Pb}$ system with the nuclear potential given in Table I).
[1] A.B. Balantekin and N. Takigawa, Rev. Mod. Phys. 70, 77 (1998).

[2] M. Dasgupta, D.J. Hinde, N. Rowley and A.M. Stefanini, Annu. Rev. Part. Sci. 48, 401 (1998).

[3] C.H. Dasso, S. Landowne and A. Winther, Nucl. Phys. A405, 381 (1983); A407, 221 (1983).

[4] K. Hagino, N. Takigawa, and A.B. Balantekin, Phys. Rev. C56, 2104 (1997).

[5] K. Hagino and A.B. Balantekin, Phys. Rev. A70, 032106 (2004).

[6] N. Rowley, G.R. Sacthler and P.H. Stelson, Phys. Lett. B254, 25 (1991).

[7] J.R. Leigh, M. Dasgupta, D.J. Hinde, J.C. Mein, C.R. Morton, J.P. Lestone, J.O. Newton, H. Timmers, J.X. Wei and N. Rowley, Phys. Rev. C52, 3151 (1995).

[8] N. Rowley, N. Grar and K.Hagino, Phys. Lett. B632, 243 (2006).

[9] H. Timmers, J.R. Leigh, M. Dasgupta, D.J. Hinde, R.C.
Lemon, J.C. Mein, C.R. Morton, J.O. Newton and N. Rowley, Nucl. Phys. A584, 190 (1995).

[10] K.Hagino and N. Rowley, Phys. Rev. C69, 054610 (2004).

[11] M.V. Andres, N. Rowley and M.A. Nagarajan, Phys. Lett. B202, 292 (1988).

[12] Muhammad Zamrun F. and K. Hagino, arXiv:0710.2753

[13] N. Rowley et al., Phys. At. Nucl., 79, 1093 (2006)

[14] S.S. Ntshangase et al., Phys. Lett. B651, 27 (2007).

[15] H. Ikezoe et al., AIP Conf. Proc., 853, 69 (2006).

[16] S. Mitsuoka, H. Ikezoe, K. Nishio, K. Tsuruta, S.C. Jeong and Y. Watanabe, Phys. Rev. Lett. 99, 182701 (2007).

[17] K. Hagino, N. Rowley and A.T. Kruppa, Comput. Phys. Commun. 123, 143 (1999).

[18] H. Esbensen, S. Landowne and H. Price, Phys. Rev. C36, 1216 (1987); 36, 2359 (1987).

[19] K. Hagino et al. (to be published).

[20] K. Washiyama, K. Hagino and M. Dasgupta, Phys. Rev. 
C73, 034607 (2006).

[21] L.R. Gasques, M. Evers, D.J. Hinde, M. Dasgupta, P.R.S. Gomes, R.M. Anjos, M.L. Brown, M.D. Rodriguez, R.G. Thomas and K. Hagino, Phys. Rev. C76, 024612 (2007).

[22] D.J. Hinde, R.L. Ahlefeldt, R.G. Thomas, K. Hagino, M.L. Brown, M. Dasgupta, M. Evers, L.R. Gasques, and M.D. Rodriguez, Phys. Rev. C76, 014617 (2007).

[23] T. Kibedi and R.H. Spears, At. Data and Nucl. Data Tables, 80, 35 (2002).

[24] S. Raman, C.W. Nestor and P. Tikkanen, At. Data and Nucl. Data Tables, 78, 1 (2001).

[25] M. Dasgupta, K. Hagino, C.R. Morton, D.J. Hinde, J.R. Leigh, N. Takigawa, H. Timmers, and J.O. Newton, Jour. Phys. G23, 1491 (1997).

[26] C.R. Morton, A.C. Berriman, D.J. Hinde, J.O. Newton, K. Hagino and I.J. Thompson, Phys. Rev. C60, 044608 (1999).

[27] E.Duek, L. Kowalsky and John M. Alexander, Comp. Phys. Comm. 34, 395 (1985)

[28] K. Hagino, N. Takigawa and S. Kuyucak, Phys. Rev. Lett. 79, 2943 (1997).

[29] K. Hagino, S. Kuyucak and N. Takigawa, Phys. Rev.
C57, 1349 (1997).

[30] R.A. Broglia and A. Winther, Heavy Ion Reactions, Vol. 84 in Frontier in Physics Lecture Notes Series (AddisonWesley, Redwood City, CA, 1991).

[31] P.R. Christensen and A. Winther, Phys. Lett. B65, 19 (1976).

[32] G.R. Sacthler and W.G. Lowe, Phys. Rep. 55, 183 (1979).

[33] J.O. Newton, R.D. Butt, M. Dasgupta, D.J. Hinde, I.I. Gontchar, C.R. Morton and K. Hagino, Phys. Rev. C70, 024605 (2004); Phys. Lett. B586, 219 (2004).

[34] W. Brenig, T. Brunner, A. Gross, and R. Russ, Z. Phys. B93, 91 (1993); W. Brenig and R. Russ, Surf. Sci. 315, 195 (1994).

[35] B.R. Johnson, J. Chem. Phys. 69, 4678 (1978).

[36] Z.H. Levine, Phys. Rev. A30, 1120 (1984).

[37] T.N. Rescigno and A.E. Orel, Phys. Rev. A25, 2402 (1982).

[38] K. Hagino, AIP Conf. Proc. 891, 80 (2006); arXiv: nucl-th/0611015.

[39] I.J. Thompson, Comp. Phys. Rep. 7, 167 (1988). 